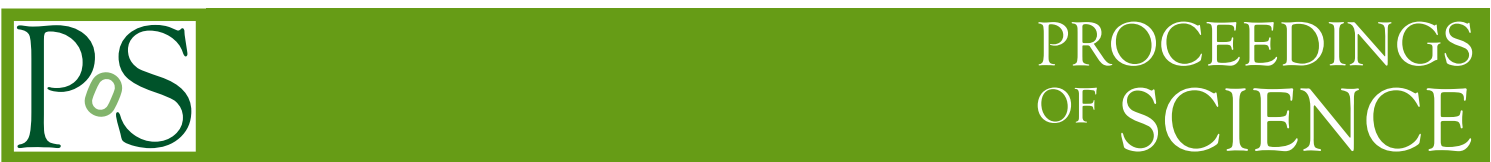

\title{
On the Quantization of Massive Superparticles
}

\author{
N.Hatcher, \\ Universidad Simón Bolivar, Departamento de Matemáticas, Apartado Postal 89000, \\ Caracas 1080-A, Venezuela. \\ E-mail: nhatcherefis.usb.ve,
}

A.Restuccia and J.Stephany*

Universidad Simón Bolívar, Departamento de Física, Apartado Postal 89000,

Caracas 1080-A, Venezuela.

E-mail: arestu@usb.ve, stephany@usb.ve

\begin{abstract}
We consider the action of the $D=11$ supermembrane wrapping a compactified sector of the target space in such a way that a non trivial central charge in the SUSY algebra is induced. We show that the dynamics of the center of mass corresponds to a superparticle in $D=9$ with additional fermionic terms associated to the central charges. We perform the covariant quantization of this system following a direct approach which introduces an equivalent action for the system which has only first class constraints allowing to obtain the space of physical states in a covariant way. The resulting multiplet contains $2^{8}$ states corresponding to a $K K B$ ultrashort multiplet.
\end{abstract}

Fifth International Conference on Mathematical Methods in Physics - IC2006 April 24-28 2006

Centro Brasilerio de Pesquisas Fisicas, Rio de Janeiro, Brazil

\footnotetext{
*Speaker.
} 


\section{Contents}

1. Introduction 2

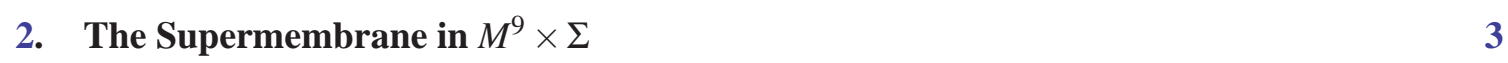

3. Compactification of the Supermembrane 4

4. The Hamiltonian for the Superparticle with central charges 5

$\begin{array}{ll}\text { 5. Conclusion } & 7\end{array}$

$\begin{array}{ll}\text { 6. Acknowledgments } & 7\end{array}$

$\begin{array}{lr}\text { A. Appendix:Dirac matrices and Central charges } & 8\end{array}$

\section{Introduction}

The theory of the supermembrane [1] is a key element in the intricate network of related systems which are expected to define the consistent non perturbative aspects of quantum superstrings. It was originally constructed as the $D=11$ extended object which propagates consistently on a supergravity background but its role in $M$-theory turn out to have more implications. It was shown in [2] that when the supermembrane is immersed in a $D=11$ Minkowski space its spectrum is continuous from $[0, \infty]$ but it is still unknown if there is a massless sector corresponding to the $D=11$ supergravity. It was also argued in Ref. [3] that the supermembrane on a compact target space should also have a continuous spectrum. In contrast the spectrum of the $D=11$ supermembrane wrapping a two cycle when the configuration space satisfies the topological condition which implies a non trivial central charge in the supersymmetric algebra, was shown to be a discrete set with finite multiplicity [4].

In this paper we focus in the low energy spectrum of the supermembrane wrapped on a two cycle by considering the covariant quantization of the associated superparticle. As we show below, the resulting action describes a massive superparticle with an additional spinorial term arising from the non trivial wrapping of the supermembrane on the two-cycle. In the massless case, as is well known, the covariant quantization of the Casalbuoni-Brink-Schwarz superparticle in $D=10$ [5] present similar obstacles related to the mixing of first and second class constraints associated with the $\kappa$-symmetry[6], as the Green-Schwarz superstring. The covariant gauge fixing of the symmetry was performed in $[7,8,9]$ by introducing an infinite tower of auxiliary fields . More recently, a new formulation was advanced in terms of pure spinors which requires a finite number of fields[10]. The dynamics of a superparticle with a central charge was first considered in [11, 12] and later in [13]. However the central charges arose in these cases from considerations different to those presented 
here. The covariant quantization of this superparticle differs from the usual massive superparticle since the central charge implies the existence of a $\kappa$-symmetry additionally to the second class constraints already present for the massive superparticle. Several formulation to quantize the superparticle with central charges were proposed [13, 14, 15]. For example in [15] a BRST charge was constructed also from a infinitely reducible set of first class constraints. Fortunately as we discuss in section 4 the structure of the system allows a direct approach in which the physical degrees of freedom are identified in a covariant way.

\section{The Supermembrane in $M^{9} \times \Sigma$}

The supermembrane action immersed in $D=11$ target space was obtained originally in Ref. [1] and is given by,

$$
\begin{array}{r}
I=-\frac{1}{8 \pi^{2}} \int d^{3} \xi\left[\sqrt{-g} g^{i j} \pi_{i}^{\mu} \pi_{j \mu}-\sqrt{-g}+i \varepsilon^{i j k} \pi_{i}^{\mu} \pi_{j}^{v} \bar{\psi} \Gamma_{\mu v} \partial_{k} \psi\right] \\
+\left[\varepsilon^{i j k} \pi_{i}^{\mu} \bar{\psi} \Gamma_{\mu \nu} \partial_{j} \psi \bar{\psi} \Gamma_{\nu} \partial_{k} \psi-\frac{i}{3} \varepsilon^{i j k} \bar{\psi} \Gamma_{\mu \nu} \partial_{i} \psi \bar{\psi} \Gamma_{\nu} \partial_{j} \psi \psi \bar{\psi} \Gamma_{v} \partial_{k} \psi\right]
\end{array}
$$

where $\psi$ is a Majorana spinor and

$$
\pi_{i}^{\mu}=\partial_{i} X^{\mu}-i \bar{\psi} \Gamma^{\mu} \partial_{i} \psi
$$

with $X^{\mu}$ the space time coordinate of the membrane. The supermembrane tension has been fixed to 1 .

We are interested in the case when the $D=11$ target space has a compactified sector admitting a minimal immersion from the base manifold $\Sigma \times R$ into it. $\Sigma$ is a Riemann surface of genus $g>0$ and $R$ corresponds to the range of the time variable $\tau$.

To be specific let us consider the case in which $\Sigma$ is a torus and the compactified target space is $M^{9} \times S^{1} \times S^{1}[16,4,17]$, although more general compactified target spaces for which there exists a minimal immersion from $\Sigma$ into the target space may also be treated along the lines we follow here [18].

The minimal immersion is constructed from the harmonic one forms over $\sigma$ denoted by $d \hat{x}^{r}$, $r=1,2$. We consider a pair of harmonic one-forms over $\Sigma$ satisfying

$$
\oint_{C_{s}} d \hat{x}^{r}=2 \pi m_{s}^{r}, \quad r, s=1,2
$$

where $m_{s}^{r}$ are integers and $C_{j}$ is a basis of the homology on $\Sigma$, together with

$$
\int_{\Sigma} d \hat{x}^{r} \wedge d \hat{x}^{s}=n A(\Sigma) \neq 0
$$

where $n=\operatorname{det} m_{s}^{r}$ and $A(\Sigma)$ is the area of $\Sigma$. The first condition implies that each $\hat{x}^{r}$ may define a map over a circle $S^{1}$. It also implies the equality in (2.4). The condition $A(\Sigma) \neq 0$ is the non trivial part of this relation.

The map from $\Sigma$ onto $S^{1} \times S^{1}$ is given, with $P_{0}$ a fixed point in $\Sigma$, by

$$
P \in \Sigma \rightarrow \int_{P_{0}}^{P} d \hat{x}^{r} \bmod \left(2 \pi n^{r}\right), r=1,2
$$


Condition (2.4) implies that the algebra of the supermembrane has a non-trivial central charge $Z^{r s}=\varepsilon^{r s} n A(\Sigma)$ with $n$ the number of times the supermembrane wraps $S^{1} \times S^{1}$. It may be shown that the above map is a local minimum of the hamiltonian of the supermembrane [19] and defines a minimal immersion from $\Sigma$ to $S^{1} \times S^{1}$ [18]. It is a solution of the supermembrane field equations which preserves one half of the original supersymmetry. The most general configuration space and hamiltonian for the supermembrane with the above base manifold and target space with a non-trivial central charge was obtained in $[4,17,16]$ and shown to have a discrete spectrum. We are interested here in the quantization of the corresponding superparticle with non-trivial central charges.

\section{Compactification of the Supermembrane}

We consider now the covariant quantization of the center of mass of the supermembrane which corresponds to a superparticle. To do so we restrict the configuration space by the following conditions,

$$
\begin{aligned}
X^{m} & =X^{m}(\tau) \quad, \quad m=0, \ldots, 8 \\
\psi & =\psi(\tau), \\
X^{r+8} & =\hat{x}^{r}(\sigma) \quad, \quad r=1,2
\end{aligned}
$$

where $\sigma^{a}, a=1,2$ denote local coordinates over $\Sigma$. On this class of configurations the supermembrane action reduces to

$$
\begin{gathered}
S \rightarrow k \int d \tau\left(e^{-1} \omega^{m} \omega_{m}-e-i \psi^{\beta}\left(\frac{1}{2} C \Gamma_{r s} \varepsilon^{r s}\right)_{\beta \gamma} \dot{\psi}^{\gamma}\right), \\
\omega^{m}=\dot{X}^{m}-i \bar{\psi} \Gamma^{m} \dot{\psi} .
\end{gathered}
$$

where $C$ is the charge conjugation matrix in $D=11$ and $\Gamma_{r s}$ is the antisymmetric product $\frac{1}{2}\left(\Gamma_{r} \Gamma_{s}-\right.$ $\Gamma_{s} \Gamma_{r}$ ). (The conventions for the $\Gamma$ matrices are given in the appendix. $C \Gamma_{r s}$ is symmetric on the spinorial indices). The constant $k$ is given by $k=\frac{n A(\Sigma)}{8 \pi^{2}}$.

In the following section we perform the covariant quantization of this system following a direct approach by introducing a new action for the superparticle with central charges with first class constraints only, that allows to obtain the space of physical states in a covariant way.

We use now a particular representation of the Dirac matrices. We consider $\gamma^{m}, m=0, \ldots, 8$ the set of Dirac matrices in $D=9$ satisfying $\left\{\gamma^{\mu}, \gamma^{\nu}\right\}=-2 \eta^{\mu \nu}$. We take as in [13],

$$
\Gamma^{m}=\left[\begin{array}{cc}
0 & i \gamma^{m} \\
-i \gamma^{m} & 0
\end{array}\right] \quad, \quad \Gamma^{9}=\left[\begin{array}{ll}
0 & i \\
i & 0
\end{array}\right] \text {. }
$$

Then we have $\left\{\gamma^{\mu}, \gamma^{v}\right\}=-2 \eta^{\mu \nu}, \mu, v=0, \ldots, 9,11$, with

$$
\Gamma^{11}=\Gamma^{0} \Gamma^{1} \ldots \Gamma^{9}=\left[\begin{array}{cc}
\mathbb{I} & 0 \\
0 & -\mathbb{I}
\end{array}\right] .
$$


The charge conjugation matrix in $D=11$ in terms of the corresponding one in $D=9$ is (see the appendix)

$$
C=\left[\begin{array}{cc}
0 & -i \tilde{C} \\
i \tilde{C} & 0
\end{array}\right]
$$

where $C^{T}=-C$ and $\tilde{C}^{T}=\tilde{C}$. Now we decompose the $D=11$ spinors in terms of the $D=9$ ones

$$
\psi=\left(\begin{array}{l}
\theta_{1} \\
\theta_{2}
\end{array}\right) .
$$

The Majorana condition $\bar{\psi}=-\psi^{T} C$ in $D=11$ implies

$$
\bar{\theta}_{A}=\theta_{A}^{T} \tilde{C},
$$

where $\bar{\theta}_{A}=\theta_{A}^{\dagger} \gamma^{0}$. The action for the superparticle with central charges reduces then to

$$
S=k \int d \tau\left(e^{-1} \omega^{m} \omega_{m}-e-i \bar{\theta}_{A} \dot{\theta}_{A}\right)
$$

where now

$$
\omega^{m}=\dot{X}^{m}-i \bar{\omega}_{A} \gamma^{m} \dot{\omega}_{A}
$$

\section{The Hamiltonian for the Superparticle with central charges}

Let us introduce here the mass parameter $m=2 k$ and the projectors,

$$
P \pm=\frac{1}{2 m}\left(m \pm \gamma^{m} p_{m}\right)
$$

which satisfy

$$
\begin{array}{ll}
P_{+} P_{+}=-\frac{1}{4 m^{2}}\left(p^{2}+m^{2}\right)+P_{+}, & P_{-} P_{-}=-\frac{1}{4 m^{2}}\left(p^{2}+m^{2}\right)+P_{-} \\
P_{+} P_{-}=P_{-} P_{+}=\frac{1}{4 m^{2}}\left(p^{2}+m^{2}\right), & P_{+}+P_{-}=\mathbb{I} .
\end{array}
$$

The conjugate momenta to $X^{m}$ are

$$
p_{m}=2 k e^{-1} \omega_{m},
$$

and the conjugate momenta to $\theta_{A}$, with $m=2 k$ are

$$
\bar{\pi}_{A}=2 i m \bar{\theta}_{A} P_{-}
$$

or equivalently

$$
\pi_{A}=-2 i m P_{-} \theta_{A} .
$$

Introducing the Lagrange multiplier $\lambda \equiv \frac{e}{4 k}$, the hamiltonian may then be expressed as

$$
\mathscr{H}=\lambda\left(p^{2}+m^{2}\right)
$$

subject to

$$
\pi_{A}+2 i m P_{-} \theta_{A}=0,
$$


which are a mixture of first and second class constraints.

The set of constraints (4.7) together with the mass shell condition $p^{2}+m^{2}=0$ are equivalent to

$$
\begin{aligned}
p^{2}+m^{2} & =0, \\
P_{+} \pi_{A} & =0, \\
P_{-} \pi_{A}+2 i m P_{-} \theta_{A} & =0 .
\end{aligned}
$$

Now (4.8) and (4.9) are first class constraints while (4.10) is a mixture of first and second class constraints.

We may then consider the set,

$$
\begin{aligned}
p^{2}+m^{2} & =0 \\
\varphi_{1}=P_{+}\left(\pi_{1}+i \pi_{2}\right) & =0 \\
\varphi_{2}=P_{+}\left(\pi_{1}-i \pi_{2}\right) & =0 \\
\Omega_{1}=P_{-}\left(\pi_{1}+i \pi_{2}\right)+2 i m P_{-}\left(\theta_{1}+i \theta_{2}\right) & =0 \\
\Omega_{2}=P_{-}\left(\pi_{1}-i \pi_{2}\right)+2 i m P_{-}\left(\theta_{1}-i \theta_{2}\right) & =0
\end{aligned}
$$

with the non trivial bracket,

$$
\left\{\Omega_{1}, \Omega_{2}\right\}=-4 i m P_{-} .
$$

We also note that the symplectic terms in the canonical action $\bar{\pi}_{A} \dot{\theta}_{A}$ may be decomposed as

$$
\bar{\pi}_{A} \dot{\theta}_{A}=\frac{1}{2} \overline{\left(\pi_{1}+i \pi_{2}\right)}\left(\dot{\theta}_{1}-i \dot{\theta}_{2}\right)+\frac{1}{2} \overline{\left(\pi_{1}-i \pi_{2}\right)}\left(\dot{\theta}_{1}+i \dot{\theta}_{2}\right),
$$

and we identify the conjugate pairs $\left(\pi_{1}+i \pi_{2}\right),\left(\dot{\theta}_{1}-i \dot{\theta}_{2}\right)$ and $\left(\pi_{1}-i \pi_{2}\right),\left(\dot{\theta}_{1}+i \dot{\theta}_{2}\right)$. We notice here that the pair $\Omega_{1}$ and $\Omega_{2}$ may be regarded as a first class constraint $\left(\Omega_{1}\right)$ and an associate gauge fixing condition $\left(\Omega_{2}\right)$. The contribution of this pair to the functional measure when taken as a pair of second class constraints or in the way proposed is exactly the same [20], [21]. With this observation we can finally define our system as a gauge system restricted by the set of first class constraints (4.11), (4.12) and (4.14). If one then would like to impose (4.15) as a partial gauge fixing condition the original set of constraints is recovered, but there is freedom to impose a different set of admissible gauge fixing conditions since the functional integral is invariant to this choice (In this case there are no gauge anomalies).

To continue we consider the partial gauge fixing conditions,

$$
\begin{aligned}
& P_{+}\left(\theta_{1}-i \theta_{2}\right)=0, \\
& P_{-}\left(\theta_{1}-i \theta_{2}\right)=0,
\end{aligned}
$$

corresponding to the symmetry generated by $\varphi_{1}$ and $\Omega_{1}$ respectively. This gauge fixing condition contributes to the functional integral with a constant factor independent of the fields. The canonical variables $\left(\pi_{1}+i \pi_{2}\right)$ and $\left(\theta_{1}-i \theta_{2}\right)$ may then be integrated out from the functional integral.

We are thus left with the canonical action, 


$$
\begin{aligned}
\mathscr{L} & =p \dot{X}+\bar{\pi}_{A} \dot{\theta}_{A}-\mathscr{H} \\
& =p \dot{X}+\frac{1}{2} \overline{\left(\pi_{1}-i \pi_{2}\right)}\left(\dot{\theta}_{1}+i \dot{\theta}_{2}\right),
\end{aligned}
$$

constrained by (4.11) and (4.13). Let us introduce the variables $\tilde{\theta}=\theta_{1}+i \theta_{2}$ and $\tilde{\pi}=\frac{1}{2}\left(\pi_{1}-i \pi_{2}\right)$. Notice that $\tilde{\theta}$ is a complex spinor which does not satisfy the pseudo Majorana condition $\bar{\theta}=\theta^{T} \tilde{C}$

We may finally consider the partial gauge fixing condition

$$
P_{+}\left(\theta_{1}+i \theta_{2}\right)=0,
$$

and perform a canonical reduction to

$$
\mathscr{L}=p \dot{X}+\overline{\left(P_{-} \tilde{\pi}\right)}\left(P_{-} \tilde{\theta}\right),
$$

subject to the mass shell condition (4.8). The space of physical states is obtained by considering superfields depending in $P_{-} \tilde{\theta}$ and not on its complex conjugate. Since we have that $P_{-}+P_{+}=1$ without using $p^{2}+m^{2}=0$ the degrees of freedom in $P_{-} \tilde{\theta}$ are exactly half of the ones in $\tilde{\theta}$. They are therefore $2^{8}$ bosonic and fermionic on-shell degrees of freedom. It corresponds as we discuss below to a $D=9 K K B$ multiplet.

\section{Conclusion}

We have covariantly quantized the $D=9$ superparticle associated to the $D=11$ supermembrane wrapped on a torus with a non trivial central charge.

The Hilbert space of states we obtained is described in terms of an on-shell superfield $\Psi\left(x^{\mu}, \tilde{\theta}_{-}\right)$. The spinorial variable $\tilde{\theta}_{-}$has 8 independent variables. The superfield has $2^{8}$ degrees of freedom that fit neatly in a $D=9$ massive supermultiplet with central charge. The general form of this central charge in $D=9$ arising from the supermembrane algebra in $D=11$ is [23]

$$
Z^{i j}=Z \delta^{i j}-\left(P_{9} \sigma^{3}-P_{10} \sigma^{1}\right)
$$

with a BPS mass given by

$$
M=\sqrt{P_{9}^{2}+P_{10}^{2}}+|Z|
$$

In this paper we have taken $P_{9}=P_{10}=0$. The multiplet that we have obtained from the quantization of the $D=9$ superparticle corresponds to a ultrashort KKB supermultiplet [23].

Due to the nature of our central charge we are studying the winding modes of the supermembrane on a torus and neglecting the Kaluza-Klein modes. As is well known [24] this states should correspond to the Kaluza-Klein modes of the IIB superstring wrapped on $S^{1}$. Our results confirm this correspondence.

\section{Acknowledgments}

This work was supported by Did-Usb grants Gid-30 and Gid-11 and by Fonacit grant G2001000712. 


\section{A. Appendix:Dirac matrices and Central charges}

We collect here some useful results about the supersymmetry algebra in $D=9$. We take the "mostly plus" signature with the Dirac matrices satisfying $\left\{\Gamma^{\mu}, \Gamma^{\nu}\right\}=-2 \eta^{\mu, \nu}$. We construct these matrices recursively. Let $\gamma^{\mu}$ be a set of Dirac matrices in $D-1$ dimensions ( $D$ even), then in $D$ dimensions we have,

$$
\Gamma^{\mu}=\left[\begin{array}{cc}
0 & S^{\mu} \\
\bar{S}^{\mu} & 0
\end{array}\right] \quad, \quad \begin{gathered}
S^{0}=\bar{S}^{0}=\mathbb{I} \quad S^{i}=\gamma^{i} \gamma^{0} \\
\bar{S}^{i}=-S^{i}=\mathbb{I} S^{D-1}=\gamma^{0}
\end{gathered} .
$$

In an even dimensional space there exist matrices $B, \tilde{B}, C$ and $\tilde{C}$ such that

$$
\begin{aligned}
& B \Gamma^{\mu} B^{-1}=-\left(\Gamma^{\mu}\right)^{*} \tilde{B} \Gamma^{\mu} \tilde{B}^{-1}=\left(\Gamma^{\mu}\right)^{*} B=\tilde{B} W \\
& C \Gamma^{\mu} C^{-1}=-\left(\Gamma^{\mu}\right)^{T} \tilde{C} \Gamma^{\mu} \tilde{C}^{-1}=\left(\Gamma^{\mu}\right)^{T} C=\tilde{B} \Gamma^{0}
\end{aligned}
$$

with $W=i^{[D / 2+1]} \Gamma^{0} \ldots . \Gamma^{D}$. In odd dimensions there exist either $(B, C)$ or $(\tilde{B}, \tilde{C})$. There exist also a matrix $\tilde{B}_{9}$ in $D=9$ and a matrix $B_{1} 1$ in $D=11$ defined by,

$$
\tilde{B}_{9}=\gamma^{0} \gamma^{1} \gamma^{3} \gamma^{5} \gamma^{7}, \quad B_{11}=\Gamma^{2} \Gamma^{4} \Gamma^{6} \Gamma^{8}
$$

such that

$$
B_{11}=\left[\begin{array}{cc}
\tilde{B}_{9} & 0 \\
0 & -\tilde{B}_{9}
\end{array}\right] \quad, \quad C_{11}=\left[\begin{array}{cc}
0 & \tilde{C}_{9} \gamma^{0} \\
-\tilde{C}_{9} \gamma^{0} & 0
\end{array}\right] .
$$

To make contact with our notation of section 3 , call that set of matrices $\Gamma_{H}^{\mu}$ they are related to $\Gamma^{\mu}$ by a unitary transformation

$$
U \Gamma_{H}^{\mu} U^{\dagger}=\Gamma^{\mu} \quad U=\left[\begin{array}{cc}
i & 0 \\
0 & \gamma^{0}
\end{array}\right] .
$$

From here follows directly equation (3.7).

The most general supersymmetry algebra in $D=9$ and $N=2$ with Lorentz invariant central charges is

$$
\left\{Q_{a i}, Q_{b j}\right\}=2 \delta i j p_{\mu}\left(\gamma^{\mu} \tilde{C}^{-1}\right)_{a b}+Z_{i j} \tilde{C}_{a b}
$$

with $Z_{i j}$ a real symmetric matrix. Note that we can write

$$
\gamma^{0} \tilde{C}=\left[\begin{array}{ll}
0 & J \\
J & 0
\end{array}\right], \quad \tilde{C}=\left[\begin{array}{ll}
J & 0 \\
0 & J
\end{array}\right], \quad J^{T}=J, J^{2}=I .
$$

Since these two matrices commute they can be simultaneously diagonalized. Then the algebra in the rest frame takes the form

$$
\left\{Q_{a i}, Q_{b j}\right\}=2 m \delta_{i j}\left[\begin{array}{cc}
J & 0 \\
0 & -J
\end{array}\right]+Z_{i j}\left[\begin{array}{ll}
J & 0 \\
0 & J
\end{array}\right] .
$$

The algebra of the 32 supercharges splits into $4,8 \times 8$ blocks. In our case $Z_{i j}=2 m \delta_{i j}$ and we find

$$
\left\{Q_{a i}, Q_{b j}\right\}=4 m \delta_{i j}\left[\begin{array}{ll}
J & 0 \\
0 & 0
\end{array}\right] .
$$

The entire representation may be obtained now as usual. We notice that half of the supersymmetries are not present and the other half build a representation of $2^{8}$ states. 


\section{References}

[1] E. Bergshoeff, E. Sezgin and P.K. Townsend, Phys. Lett.189B (1987) 75; E. Bergshoeff, E. Sezgin and P.K. Townsend, Ann. Phys. 185 (1988) 330.

[2] B. de Witt, M. Lüscher and H.Nicolai, Nucl. Phys. B320 (1989) 135

[3] B. de Wit, K.Peeters and J.C.Plefka Nucl. Phys. Proc. Suppl. 62, (1998), 40

[4] L.Boulton, M.Garcia del Moral and A.Restuccia Nucl. Phys. B671 (2003) 343.

[5] L.Brink and J.H.Schwarz Phys. Lett. 100B, (1981), 310; R.Casalbuoni, Phys. Lett.62B (1976), 49.

[6] W.Siegel, Phys. Lett. 195B, (1987), 369.

[7] R.Kallosh, Phys. Lett. 195B, (1987), 369.

[8] A.Diaz and J.Zanelli, Phys. Lett. 202B, (1988), 347.

[9] A.Restuccia and J.Stephany, Phys. Rev. D47, (1993), 3437; Phys. Lett. 343B (1995), 147.

[10] N.Berkovits, J.High Energy Phys. 0209 (2002) 051.

[11] J.A. de Azcárraga and J.Lukierski Phys. Lett. 113B, (1982), 170; J.A. de Azcárraga and J.Lukierski Phys. Rev. D28, (1983), 1337.

[12] V.Aldaya and J.A. de Azcárraga Phys. Lett. 121B, (1983), 331.

[13] M.Huq, Phys. Lett. 205B (1988) 4791.

[14] A.Frydryszak and J.Lukierski Phys. Lett. 117B, (1982), 51.

[15] Ž. Antunović and M.Blagojević, Nucl. Phys. B363 (1991) 622.

[16] L.Boulton, M.Garcia del Moral, I.Martín, A.Restuccia, Class. Quan. Grav. 19, (2002), 2951.

[17] M.Garcia del Moral, A.Restuccia, Phys. Rev. D66, (2002), 045023.

[18] J.Bellorín and A.Restuccia, hep-th/0312265.

[19] I.Martín, J.Ovalle and A.Restuccia, Phys. Lett. B472, (2000), 77, hep-th/9909051.

[20] R.Gianvittorio, A.Restuccia and J.Stephany Mod. Phys. Lett. A6 (1991) 2121.

[21] A.Restuccia and J.Stephany, Phys. Lett. 305B, (1993), 348.

[22] M.J.Duff, T.Inami, C.N.Pope, E.Sezgin and K.S.Stelle, Nucl. Phys. B297 (1988) 515.

[23] M. Abou-Zeid, B. de Wit, D. Lüst, H. Nicolai, Phys. Lett. 466B, (1999), 144.

[24] J. H. Schwarz Phys. Lett. 360B, (1995), 13. 